\title{
QoS Management System of Smart Grid Communication Network Based on SDN Technology
}

\author{
Meng Yin ${ }^{1}$, Min $\mathrm{Zhu}^{2}$,Yong-en Wang ${ }^{3}$ and Chenbo Yuan ${ }^{2}$ \\ 1. China Mobile Group Design Institute Co., Ltd Beijing Branch \\ 2. State Grid Meishan Electric Power Supply Company, sichuan, China \\ 3. China Electrical Power Research Institute, Beijing , China
}

Keywords: Smart Grid; Internet Protocol Version 6; Software Defined Network technology; Quality of Service; Border router

\begin{abstract}
Smart grid components are free and secure for exchanging data that requires a good data communication network to support, the next generation of Internet technology applications become an inevitable trend. Internet Protocol Version 6 as the core of the next generation of Internet construction, with its own advantages to promote the development of smart grid information. In view of the complexity of the future power grid business, the traditional routing mechanism load serious problems, The article proposes SDN technology to design a QoS management system which can allocate and allocate network traffic to the underlying terminal network, And then use the border router to the priority of each terminal sort and send the results to the core router, to achieve the highest transmission efficiency of each terminal QoS, making the power communication network service quality improvement. At last, this paper tests the SDN programming interface of the video data transmission service of the smart grid, and the boundary router QoS management system simulation.
\end{abstract}

\section{Introduction}

The future of power grid information and communication network operation mainly rely on the next generation of Internet technology, IPv6 as the core construction area of the next generation of Internet development will be widely used. The features of the next generation Internet, like huge address space, efficient media exchange, forwarding capabilities, assurance of supporting end-to-end service quality, protect the smart grid operation and construction. SDN technology as a new network innovation architecture is being studied in various fields, openflow 1.3 version of the proposed protocol, the control and forwarding layer routing loosely coupled to enhance the IPv6 header field recognition function, so that SDN network is possible to implement a centralized QoS management system. In this paper, SDN technology is used to design a switch based on the openflow protocol and interconnection with the border router to realize the network QoS management system. Through this management system, the QoS guarantee of the next generation Internet between the terminals of the power grid is realized. 


\section{Introduction to the system}

IPv6 (Internet Protocol Version 6) is the next generation IP protocol used to replace the current version (IPv4). IPv6 has the following advantages: IPv6 has a larger address space, the length of IP addresses in IPv6 is 128, that is, the maximum number of addresses is 2128; IPv6 uses a smaller routing table, IPv6 address allocation follows the principle of clustering, which makes the router can use a record in the routing table to represent a subnet, reduce the length of the routing table in the router, and improve the speed of the router forwarding packets. IPv6 adds enhanced multicast support and convection support, which makes the network multimedia applications have a lot of opportunities for development, provides the quality of service (QoS) control with a good network platform; allow expansion, if the new technology or application needs, IPv6 allows the agreement to expand [1].

Software Defined Network (SDN) technology is a new type of network architecture, the core technology openflow separating the network equipment control surface and data surface, in order to achieve the flexible control of network traffic, provide a good platform for the core network and application Innovation to. SDN is applied in the next generation of Internet technology, the basic idea is to separate the current IP network interconnection node to which determine how the message forwarding complex logic from the switch / router and other devices to control data forwarding rules by hardware with the method of software programming, and ultimately to achieve the purpose of free flow control.

Border router is responsible for packet classification, monitoring and scheduling, it's implementation is based on adding a corresponding router on the corresponding module or program. Specific features are above:

(1) Identify the packets and assign them to a specific stream, assign the corresponding QoS type to the flow, work with the packet scheduler, classify the packets using the TC field of the IPv6 packet header, and set the priority in the packet, effectively avoid the type of restrictions.

(2) communication control, receiving programming scheduling in the router interface, control of the communication.

Application Programmable Interface: The current application of programmable interface to achieve the following are the following, Nicira team developed the first open source SDN controller NOX and its follow-up version of POX using Python to define the application programmable interface; Big switch team developed Flood Light controller and the latest Open Day Light controller provide a standard REST-style northbound interface. REST API has been widely used in SDN interface design with its flexible ease of use. In this paper, the implementation of REST style Web service will be used to divide the controller resources, determine the URI of the resource, design the operation method of the resource, and finally implement the application programmable interface with the guarantee of service quality.

\section{Key Technologies}

\subsection{Openflow Protocol}

The openflow protocol is the foundation of SDN's control and forwarding separation. The Open Networking Fundation (0NF) has released multiple openflow versions since October 2009, making the SDN of the openflow protocol a new standard for the network. The openflow protocol defines the interface standard between the SDN controller and the switch, as well as the communication standard between the controller and the switch [9].

(1) flow control 
The switch port can be configured with one or more queues; packets are processed by a flow table, enqueue actions are performed, and groups can be added to the specified queue. The port queue has the maximum and minimum rate limits, and the packet forwarding rate mapped to the queue is controlled accordingly.

\begin{tabular}{ccc} 
TABLE I. SDN SERVICE QUALITY AsSURANCE SUPPORT TECHNOLOGY \\
\hline QoS capability & $\begin{array}{c}\text { Switch } \\
\text { components }\end{array}$ & $\begin{array}{c}\text { The type of key } \\
\text { message }\end{array}$ \\
\hline flow control & queue & $\begin{array}{c}\text { Queue Configuration } \\
\text { Messages }\end{array}$ \\
\hline Distinguish services & Meter queue & $\begin{array}{c}\text { Meter Modification } \\
\text { Messages }\end{array}$ \\
\hline MPLS & Action table & $\begin{array}{c}\text { Push-Tag / Pop-Tag, } \\
\text { Push-MPLS }\end{array}$ \\
\hline Routing control & Flow table & Flow \\
& & TableModification \\
& & Messages \\
\hline
\end{tabular}

\section{(2) differentiated services}

The openflow version 1.3 adds a meter to the switch, which not only tests the forwarding rate of the packet, but also controls the forwarding rate of the packet. Meters are used in conjunction with port queues to implement complex QoS frameworks such as differentiated service models.

(3) MPLS (Multi-Protocol Label Switching)

MPLS can improve the performance of IP packet flow through fast forwarding and improve the support capability of real-time services.

(4) routing control

The flow table is the only forwarding rule in the data layer of the SDN network, and the flow table is developed by the controller and centrally managed. The routing is dependent on the data layer forwarding rules. Therefore, the controller is the core of routing control in SDN networks.

This paper uses QoS resources such as switch flow table and queue to provide QoS resource control capability for the application layer in the application interface, so that the network application can flexibly call the service quality assurance resources. Figure 1 shows the openflow of data packet processing mechanism.

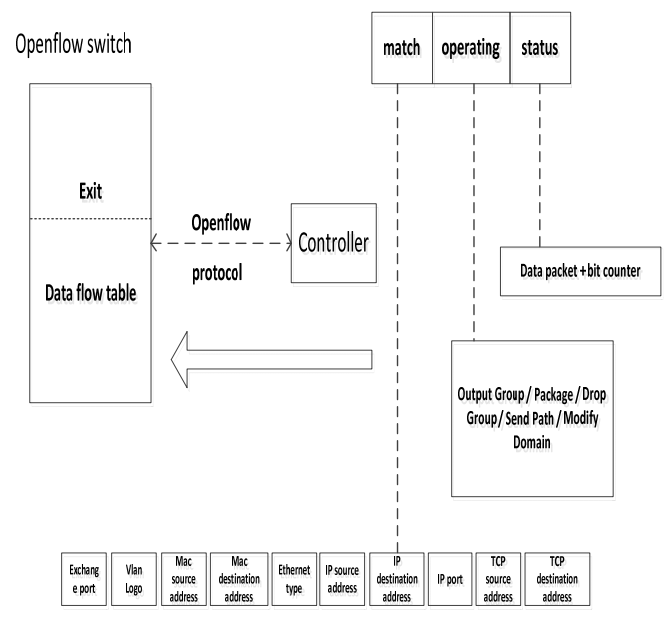

Fig. 1. The processing mechanism of a openflow data packet 


\subsection{QoS technology}

QoS (Quality of Service) refers to a network can use a variety of basic technologies for the specified network communication to provide better service capabilities, it is a network security mechanism which is used to solve the problem of network delay and congestion. After the proposed openflow protocol, the control and forwarding layer routing loosely coupled the characteristics of the SDN network to achieve centralized QoS management system possible. In this paper, according to the idea of differentiated service, according to the controller application interface to provide the flow table, queue operation function, to achieve shunt, strategy, queue and scheduling processing flow, improve the SDN network service assurance capabilities, with good flexibility and scalability ${ }^{[10]}$.

\subsection{SDN technology}

SDN is a new type of network innovation architecture, its core technology openflow through the network equipment control surface and data surface separation, the control will be transferred to the computing device, through the upper application and service on the underlying facilities to abstract, the network as a logical or virtual entity. So as to achieve the flexible control of network traffic for the core network and application innovation provides a good platform. SDN network architecture is shown in Figure 2. Network intelligence is focused on the software-based SDN controller, which can manage the entire network [4].

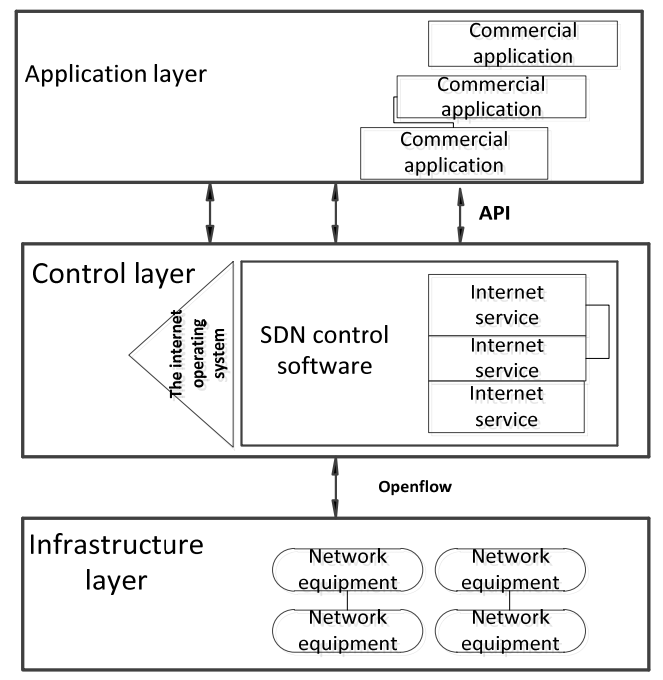

Fig. 2. SDN model architecture

\subsection{SDN programmable interface technology}

REST API has been widely used in SDN interface design with its flexible ease of use. In this paper, the implementation of REST style Web services will be used to divide the controller resources, determine the URI of the resource (unified resource identifier), design the operation method of the resource, and finally implement the application programmable interface with the guarantee of service quality. Quality of service interface to ensure the resolution of three issues of topology management, QoS resource control and QoS strategy analysis. The basic requirement for service quality assurance is to develop QoS policies based on network status. Before the policy is developed, the current resource status of the network must be obtained first, and the controller can control the resource usage in the network through the topology management function. Therefore, 
the application interface should first have a topology management API (application programming interface). Second, the application needs to control the flow table, queue, meter and other resources, and real-time access to the status of these resources, in addition to the application interface also need to provide QoS resource control API. Finally, the application interface must provide the policy analysis API, the network application to develop QoS strategy to resolve, and accurately issued to the controller [5].

\section{System Architecture}

In the SDN centralized network environment, the use of openflow switch to provide QoS support technology, combined with the controller application programmable interface technology, the use of REST technology design and implementation of the service quality assurance application programmable interface, in the preparation of JAVA class programming, As the basic class of the interface implementation, the control logic of the interface is implemented. the topology manager, policy manager, flow manager, queue manager and meter manager are used to manage the QoS resources. Since this QoS management system is focused on the different services of the future power grid Type management, so the different business has a different interface programming procedures, so the article on the SDN control interface programming to write a detailed description. Figure 3 displays the SDN-based QoS application interface design.

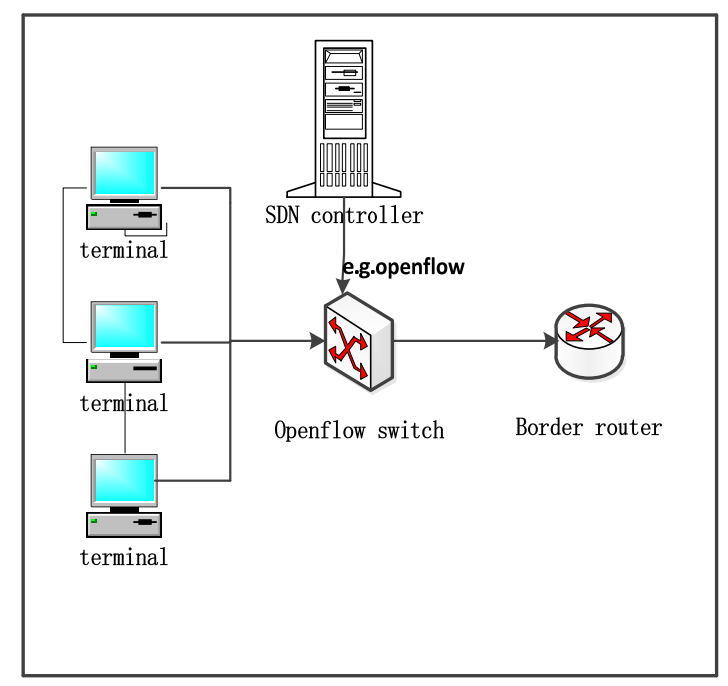

Fig. 3. SDN-based QoS application interface design

For the different data transmission scenarios and services of the smart grid end-to-end, the QoS resource control operation provided by the SDN controller interface is used to realize the differentiated service in the SDN network based on the idea of differentiated service. The use of openflow switch in the bottom of the power grid to form a terminal of a local area network, the use of SDN controller set the quality of service parameters, the terminal LAN network resources for scheduling. This paper first based on the openflow switch flow table, queue, meter and other service quality assurance technology, the use of REST style network service technology, design and implementation of a service quality assurance controller application programmable interface, in accordance with the idea of differentiated services, according to data Streaming, strategy development, entry and scheduling of the processing flow, based on the programmable interface QoS service program to improve the application of service quality ${ }^{[3]}$. 


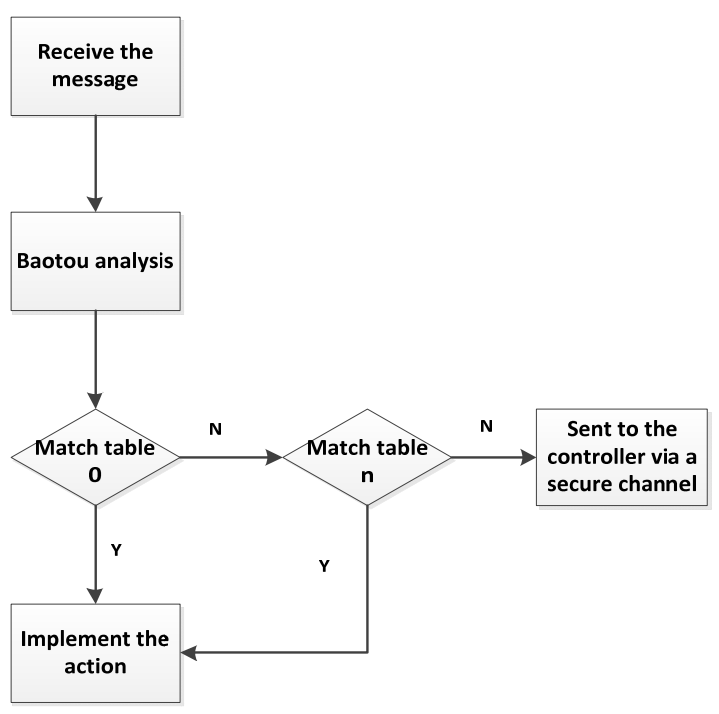

Fig. 4. Flow chart for packet processing in openflow switches

When an OpenFlow switch receives a packet, it matches the entries in its locally saved flow table according to the priority, and takes the matching entry with the highest priority as the matching result, and according to the corresponding action to the packet To operate. At the same time, once the match is successful, the corresponding counter will be updated; and if the matching entry cannot be found, the packet will be forwarded to the controller, as shown in Figure 4.

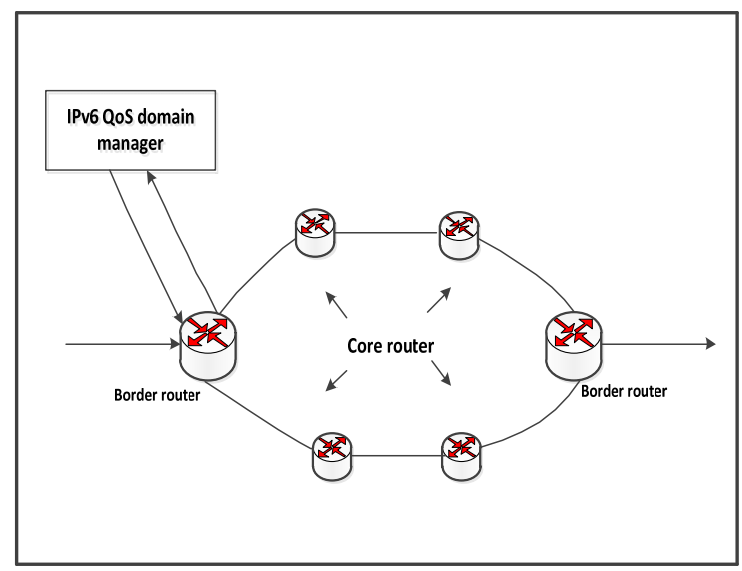

Fig. 5. structure of the five-border router QoS management system

Based on the SDN model, the QoS management system uses the border router to compose the system structure based on the border route. The source node communicates with the openflow switch to determine the priority backbound border router to send the QoS request. After receiving the request, the border router has theIPv6 QoS manager, the contact request decides whether to approve the request, and then sends the manager's response back to the openflow switch. The border router classifies, dispatches and supervises the packet, and sends it in line with its TC domain settings and the communication specification set by the QoS manager. The core router uses GFI to make forwarding decisions by the manager's communication settings. The QoS manager of each domain only manages the border routers in the domain without participating in the final communication and is responsible for coordinating with the neighboring domains to ensure the scalability of the system. The core router only uses the GFI to make forwarding decisions, the use of the longest matching efficiency is higher, is conducive to reducing the delay [11]. 


\section{Interface Testing And System Simulation}

The article uses a server and a number of hosts to build a small SDN simulation network experimental environment, the experiment was installed in three communication hosts iperf client and server side, uninterrupted transmission of UDP packets, analog power network terminal two users at the same time receive different clarity Degree of video application scenarios, the two users to provide different quality of service guarantee. Statistics client real-time bandwidth, packet loss rate, jitter, measure the quality of the client's service.

Two users in the service quality assurance and no service quality assurance before and after the two testing process, the link transmission real-time bandwidth, jitter, packet loss rate statistical results are shown in Figure 6.

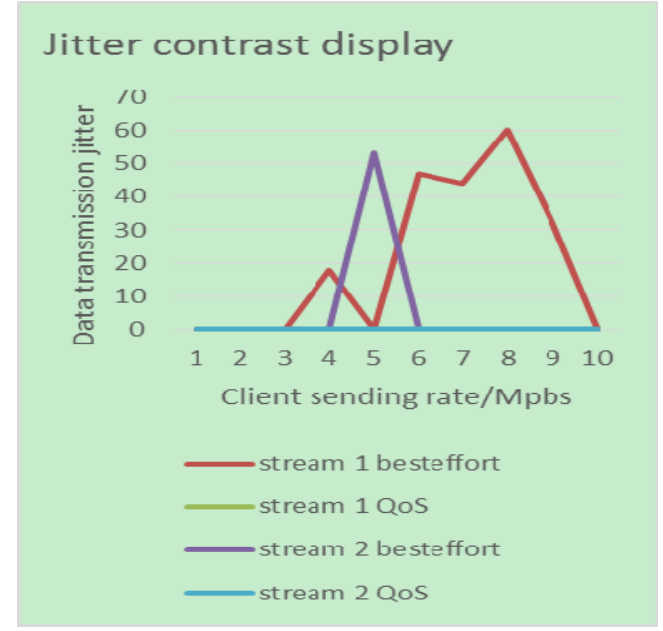

(a) Jitter

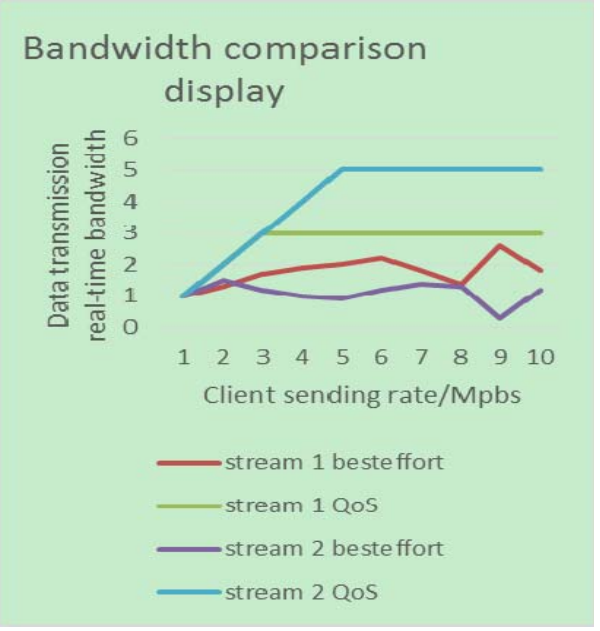

(b) Bandwidth

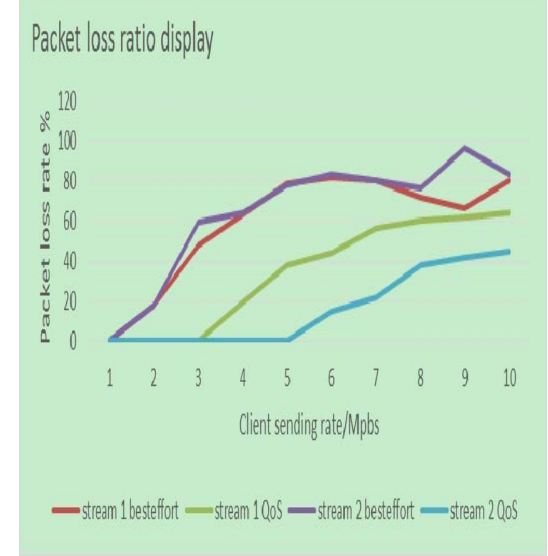

(c) packet loss rate

Fig. 6. Comparison of results

From the results can be seen, before and after the two bandwidth, jitter, packet loss rate of the situation has obvious differences. Through the application interface to add quality of service guarantee, the link transmission is significantly more stable. This fully illustrates that network applications based on application interfaces can provide good network traffic scheduling, and the design interface get good programmability.

In order to investigate the system performance, verify its superiority and shortcomings, building the NS-2 simulation environment in the Linux platform, the case of QoS management domain, a 
number of communication flow scenarios to experiment. Topological structure shown in Figure 7 , flow parameters set in Table II.

Table II. flow parameter settings

\begin{tabular}{lcccc}
\hline flow number & $\begin{array}{c}\text { Openflow } \\
\text { source node }\end{array}$ & priority & $\begin{array}{c}\text { Constant } \\
\text { rate } / \mathbf{M b} \cdot \mathbf{s}^{-1}\end{array}$ & $\begin{array}{c}\text { Change rate } \\
/ \mathbf{M b} \cdot \mathbf{s}^{-1}\end{array}$ \\
Flow1 & $\mathrm{S} 1$ & 15 & 0.50 & 0.70 \\
Flow2 & $\mathrm{S} 2$ & 12 & 0.25 & 0.35 \\
Flow3 & $\mathrm{S} 3$ & 8 & 0.25 & 0.25 \\
\hline & & & & \\
\end{tabular}

Fig. 7. QoS management system simulation topology

When the three openflow switch source nodes S1, S2, and S3 respectively transmit data streams Flow1, Flow2 and Flow3 at a constant rate of $0.5 \mathrm{Mb} / \mathrm{s}, 0.25 \mathrm{Mb} / \mathrm{s}$ and $0.25 \mathrm{Mb} / \mathrm{s}$ at the priority of 15, 12 and 8, respectively, The end-to-end delay of the destination node is 13.49 $\mathrm{ms}, \quad 13.81 \mathrm{~ms}$ and $18.42 \mathrm{~ms}$, respectively. If the priority of 12 flow increased by $40 \%$, the network bandwidth is not enough to allocate, congestion cannot be avoided, the results shown in Table 3, the delay change shown in Figure 7 . If the priority of 15 flow increased by $40 \%$, the results shown in Table III. When the number of nodes sending packets increases and the priority level increases, the average end-to-end delay increases, but the packet loss rate does not increase significantly.

Table III. Flow 2 increased by $40 \%$ of the average delay and packet transmission statistics

\begin{tabular}{|c|c|c|c|c|c|c|c|}
\hline \multirow{2}{*}{$\begin{array}{c}\text { Flow } \\
\text { number }\end{array}$} & \multirow{2}{*}{ priority } & \multirow{2}{*}{$\begin{array}{c}\text { Average } \\
\text { delay /ms }\end{array}$} & \multirow{2}{*}{$\begin{array}{c}\text { number of } \\
\text { Sending } \\
\text { packets }\end{array}$} & $\begin{array}{c}\text { Number of } \\
\text { packs }\end{array}$ & percentage & $\begin{array}{c}\text { Number } \\
\text { of packs }\end{array}$ & percentage \\
\hline Flow1 & 15 & 15.19 & 3421 & 0 & 0 & 0 & 0 \\
\hline Flow2 & 12 & 59.21 & 2395 & 272 & 11.4 & 230 & 9.6 \\
\hline Flow3 & 8 & 113.24 & 1711 & 0 & 0 & 115 & 9.8 \\
\hline merge & & & 6987 & 272 & 11.4 & 345 & 19.4 \\
\hline
\end{tabular}

Through the above simulation, we can see that in the IPv6 network, the QoS management domain can provide excellent end-to-end delay guarantee and low packet loss rate, especially for high priority traffic flow. When congestion occurs in the network, Priority traffic flow; when the rate of change occurs in the communication process, the low priority flow is more serious, but the high priority flow can get better QoS guarantee.

\section{References}

[1] Zhang Wei, Yan Li. The next generation of Internet technology in the smart grid application [J], Shanxi Electric Power Company credit branch, power system communication, 2012, vol 242,No.33. 
[2] Duan Hongchao, Wang Xingwei, Huang Min.IPv6 Multicast QoS Routing Mechanism [J] .Computer Science and Exploration, 2015,9 (8): 935-944.

[3] Zhang Jincang, Huang Yueying, Zhao Shoukai. Research on improved QoS model based on network calculus [J]. Journal of Southwest China Normal University: Natural Science Edition, 2014,39 (3): 1-8.

[4] Zhang Shunmiao, Zou Fumin. Review of Software Definition Network [A]. Fujian Institute of Engineering, Computer Application Research, 2013, Vol.30, No. 8.

[5] Design and Implementation of SDN Northbound Interface Supporting Customized QoS Service [J]. Journal of Nanjing University of Posts and Telecommunications, 2016, Vol. 26, No.11.

[6] NAHRSTEDTK, WICHADAKULD, XUD.Distributed QoS Compilation and Runtime Instantiation [C]//Quality of Service, 2000.IWQOS.2000 Eighth International Workshop on, IEEE, 2000:1 9 8-20 7.

[7] STOICA Ion.Stateless Core:A Scalable Approach for Quality of Service in the Internet:Winning Thesis of the 2001 ACM Doctoral Dissertation Competition [M].New York:Springer US, 2004.

[8] Wang Hongxin. QoS based on IPv6 flow tag [D]. Shenyang: Liaoning University, 2011. 